\title{
OPINIONES DE FAMILIAS VULNERABLES SOBRE PROMOCIÓN DE LA SALUD BUCAL INFANTIL: UN ANÁLISIS DE CONTENIDO ${ }^{1}$
}

\author{
VIEWS OF VULNERABLE FAMILIES ON PROMOTING CHILDREN'S \\ ORAL HEALTH: A CONTENT ANALYSIS
}

\section{OPINIÓES DE FAMÍLIAS VULNERÁVEIS NA PROMOÇÃO DA SAÚDE ORAL INFANTIL: UMA ANÁLISE DE CONTEÚDO}

\author{
Maggie Campillay Campillay* \\ Fabián Araya Galleguillos** \\ Ana Calle Carrasco*** \\ Pablo Dubó Araya**** \\ Verónica Anguita MaCkaY***** \\ ANDrea CARrizo Zamorano ${ }^{* * * * * *}$
}

\section{RESUMEN}

Objetivo: El propósito de este estudio fue interpretar las opiniones de familias que participaron en el programa de promoción de la salud bucal "Sembrando Sonrisas" en la Región de Atacama. Material y Método: Estudio descriptivo-interpretativo que utilizó metodología cualitativa y análisis de contenido. Se analizaron 294 opiniones expresadas por 194 familias, en el cuaderno viajero del programa piloto realizado el año 2017 en doce jardines públicos repartidos en cuatro comunas diferentes de la región. El análisis de contenido se realizó con apoyo del programa Atlas.ti . Resultados: En la primera fase se construyeron 25 categorías y en una segunda fase emergieron 8 categorías teóricas, destacando: actitudes de los padres sobre la salud bucal en sus hijos(as), conocimiento sobre salud bucal, relación lúdica con la higiene bucal, relación entre imagen física y salud bucal,

${ }^{1}$ Este artículo es resultado del Convenio de colaboración en investigación entre la SEREMI de Salud y la Universidad de Atacama; no existieron fuentes de apoyo económico.

*Dra. en Enfermería, Departamento de Enfermería, Facultad Ciencias de la Salud, Universidad de Atacama, Copiapó, Chile. ORCID: https://orcid.org/0000-0002-4054-1595, Email: maggie.campillay@uda.cl Autor de correspondencia.

**Magíster en Salud Pública, Departamento de Medicina, Facultad de Medicina, Universidad de Atacama, Copiapó, Chile. ORCID: https://orcid.org/0000-0002-6225-4490,Email: fabian.araya@uda.cl Autor de correspondencia.

${ }^{\star * \star}$ Magíster en Terapia manual ortopédica, Departamento de Kinesiología, Facultad de Ciencias de la Salud, Universidad de Atacama, Copiapó, Chile. ORCID: https://orcid.org/0000-0002-8099-1486, Email: ana.calle@uda.cl

****Enfermero, Departamento de Enfermería, Facultad Ciencias de la Salud, Universidad de Atacama, Copiapó, Chile. ORCID: https://orcid.org/0000-0001-6918-3659, Email: pablo.dubo@uda.cl

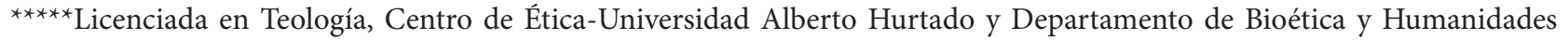
Médicas, Facultad de Medicina, Universidad de Chile, Santiago, Chile. ORCID: https://orcid.org/0000-0001-6078-8723, Email: vanguita@uahurtado.cl

*****Enfermera, Programa Magíster de Investigación en Metodologías Cualitativas en Salud, Facultad Ciencias de la Salud, Universidad de Atacama, Copiapó, Chile. ORCID: https://orcid.org/0000-0001-5915-0984, Email: andrea.carrizo.14@ alumnos.uda.cl 
entre otras. Conclusiones: La estrategia educativa contribuye a disminuir brechas de conocimiento en salud bucal de padres y madres de sectores vulnerables, y aporta a mayor justicia social en cuanto mayor acceso al cuidado bucal, pero se requiere de acompańamiento continuo de personal capacitado.

Palabras clave: Promoción de la salud; Salud preventiva; Salud bucal; Vulnerabilidad; Determinantes sociales de la salud; Familia.

\begin{abstract}
Objective: The purpose of this article is to evaluate the opinions of families that participated in the oral health promotion program "Sembrando sonrisas" (Sowing smiles) in the Atacama Region in northern Chile. Materials and Methods: Descriptive-interpretative study using qualitative methodology and content analysis. We analyzed 294 opinions expressed by 194 families in the class project book called "cuaderno viajero" of the pilot program, carried out in 2017 in twelve public kindergardens of four different cities. The content analysis was carried out with the support of the Atlas.ti ${ }^{\ominus}$ software. Results: 25 categories were established in the first phase and 8 theoretical categories emerged in a second phase, of which the following stand out: parental attitudes that favor oral health in their children, knowledge about oral health, playful relationship with oral hygiene, relationship between physical image and oral health, among others. Conclusions: The educational strategy contributes to reducing gaps in knowledge of oral health among parents from vulnerable sectors, and contributes to greater social justice in terms of greater access to oral care, but requires continuous monitoring by trained personnel.
\end{abstract}

Key words: Health promotion; Preventive health; Oral health; Vulnerability; Social determinants of health; Family.

\title{
RESUMO
}

Objetivo: O objetivo deste artigo foi interpretar a opiniáo das famílias que participaram do programa de promoçáo da saúde bucal "Semeando sorrisos" na Regiáo de Atacama. Material e Método: Estudo descritivointerpretativo que utilizou metodologia qualitativa e análise de conteúdo. Foram analisadas 294 opiniôes expressas por 194 famílias, no caderno itinerante do programa piloto realizado em 2017 em doze creches públicas de quatro cidades diferentes na regiáo. A análise de conteúdo foi realizada com o apoio do programa Atlas.ti ${ }^{\oplus}$ Resultados: Na primeira fase foram construídas 25 categorias e na segunda fase emergiram 8 categorias teóricas, destacando: atitudes dos pais sobre a saúde bucal de seus filhos(as), conhecimentos sobre saúde bucal, relação lúdica com a higiene bucal, relação entre imagem física e saúde bucal, entre outras. Conclusóes: A estratégia educativa contribui para a redução das lacunas de conhecimento em saúde bucal de pais e mães de setores vulneráveis e contribui para maior justiça social em termos de maior acesso à higiene bucal, mas é preciso acompanhamento contínuo de pessoal capacitado.

Palavras-chave: Promoção da saúde; Saúde preventiva; Saúde bucal; Vulnerabilidade; Determinantes sociais da saúde; Família.

Fecha de recepción: 21/09/2020

Fecha de aceptación: 04/01/2021

\section{INTRODUCCIÓN}

Aproximadamente el $90 \%$ de niños(as) en edad escolar sufren enfermedad dental, alterando funciones bucales esenciales e influyendo en su autoestima. Evitar o retardar la aparición de este problema en la niñez elimina la necesidad de someterlos a extensos procesos de rehabilitación, y a que sus familias asuman elevados costos económicos ${ }^{(1)}$. La Organización Mundial de la Salud (OMS) ha llamado a priorizar acciones sobre la población infantil para mantener la dentición 
saludable por el mayor tiempo posible y reducir desigualdades injustas que determinan la mala salud bucal. Desde esta perspectiva, la política nacional de salud bucal de la infancia prioriza la promoción de la salud y prevención de la enfermedad bucal.

En Chile esta estrategia se ha implementado en jardines infantiles públicos con altos índices de vulnerabilidad, a través de un fuerte componente educativo a cargo del equipo interdisciplinario, educadores de párvulos y la comunidad. El Cuaderno Viajero es parte de las actividades pilotos del programa "Sembrando Sonrisas" del Chile Crece Contigo, realizada en jardines infantiles públicos, que conforma una serie de prestaciones para el desarrollo de la infancia. Tiene como eje la promoción de la salud y prevención específica, y es ejecutado por los Centros de Salud Familiar $(\mathrm{CESFAM})^{(2)}$. En otros países, esta estrategia ha demostrado eficiencia y sostenibilidad en el tiempo, al contribuir en la formación de buenos hábitos de salud bucal en etapas tempranas de la niñez ${ }^{(2-4)}$.

La inequidad en el acceso a la atención dental es una de las grandes barreras para mantener buena salud bucal y ampliar la conciencia del cuidado, realidad que se observa cuando los países desarrollados muestran menor prevalencia de enfermedad bucal que países en vías de desarrollo ${ }^{(5)}$. Existe gran daño dental en grupos de niños(as) y adolescentes en condiciones de pobreza, transformando la enfermedad bucal en un importante problema de salud pública a nivel global. Esta distribución desigual se asocia a inequidades socioeconómicas que vulneran el derecho a la salud de niños(as) por no acceder a la atención dental ${ }^{(6)}$, generando exclusión ${ }^{(7)}$.

En Chile se han descrito inequidades en el acceso a servicios dentales relacionadas con ruralidad, migración, bajo nivel educacional de los padres y falta de acceso a agua potable fluorada. Esta realidad coincide con estudios internacionales que identifican al entorno del hogar como una de las variables más importantes en la aparición de caries infantil $^{(8,9)}$. Desde la perspectiva de la infancia, sería una expresión de que los determinantes sociales de la salud se definirían como las circunstancias sociales en que los cuidadores viven, trabajan e influyen en la salud, crecimiento y desarrollo de niños $(\mathrm{as})^{(10)}$. Frente a estas circunstancias, estudios en neurociencia han advertido que niños(as) expuestos a condiciones de vida adversas son más susceptibles a una variedad de problemas de salud durante su adultez ${ }^{(11)}$. Por tanto, estas injusticias sociales deben ser visibilizadas y abordadas a través de políticas públicas para mejorar las condiciones de vida de la población, y para que, independiente de su condición socioeconómica, todo niño(a) pueda tener las mismas oportunidades y lograr desarrollar su máximo potencial ${ }^{(10)}$.

Los costos de rehabilitar la población afectada y desatendida en salud bucal, está muy por sobre los costos asociados a realizar acciones de prevención específicas y de promoción de la salud que, especialmente en etapas tempranas de la niñez, buscan alcanzar mayores habilidades para el autocuidado ${ }^{(12)}$. A pesar de los beneficios de la costo/eficacia demostrada por la promoción de salud y programas de prevención primaria, las intervenciones en salud en Chile siguen construyéndose a partir de la lógica biomédica ${ }^{(13)}$. La promoción de la salud se define como toda actividad salutogénica que aumenta el control y mejora la salud de las personas, aporta a la visión holística, favorece la equidad, participación y empoderamiento de las personas y la intersectorialidad ${ }^{(10,14)}$. Este enfoque es el necesario para enfrentar la mala salud bucal en la infancia, mantenida por la relación del bajo nivel socioeconómico con inadecuadas conductas en salud, exposiciones ambientales y falta de acceso a la atención odontológica ${ }^{(15)}$.

Estos aspectos han sido recogidos en el programa de salud odontológico de la atención primaria de salud chileno, con resultados variados: por un lado, diferencias negativas en salud bucal de niños(as) que viven en sectores rurales de otros/as que viven en zonas urbanas ${ }^{(1)}$ y por otro, disminución en la prevalencia de caries en escolares de 6 a 12 años, con mejores resultados en niños que niñas ${ }^{(16)}$.

En este contexto, se sabe que la alfabetización en salud oral de los cuidadores está asociada a buenos niveles de salud bucal en los hijos(as) ${ }^{(17)}$ y que la estrategia individual no ha tenido tan buenos resultados ${ }^{(18)}$ como las estrategias educativas grupales y comunitarias con demostrada efectividad por la participación de la familia ${ }^{(3)}$. Estas intervenciones han sido utilizadas en distintos países y aceptadas a nivel mundial teniendo el potencial de facilitar cambios y actitudes en favor de la salud dental ${ }^{(10,17)}$.

Basado en lo planteado, esta investigación tuvo el propósito de interpretar opiniones de las familias que participaron en la actividad "Cuaderno Viajero: 
Familias que Siembran Sonrisas". Esta estrategia es parte del componente educativo del programa Sembrando Sonrisas del Plan Nacional de Salud Bucal Infantil de Chile, que buscó fortalecer la promoción de hábitos saludables y acciones preventivas dentales en niños(as) que asisten a jardines infantiles públicos vulnerables en la Región de Atacama-Chile el 2017.

La intervención, realizada por el equipo de salud, duró un año e incluyó exámenes dentales, aplicación de barniz de flúor dos veces al año, entrega de pasta y cepillo dental y talleres educativos a educadoras de párvulos y familias. Durante esta intervención, las familias expresaron sus opiniones en un cuaderno sobre prácticas en salud bucal y autocuidado de los nińos(as), permitiendo intercambiar experiencias y costumbres entre ellas. El análisis de este material fue solicitado por la SEREMI de Salud de Atacama, para contar con un diagnóstico regional, y contribuir a mejorar la estrategia educativa realizada por los equipos de Atención Primaria de la Salud y el intersector involucrado.

El valor social de este trabajo consideró fortalecer o desestimar la estrategia de salud bucal piloto en la región, con el fin de tomar las mejores decisiones sanitarias que beneficiaran a los sectores más vulnerables de la población.

\section{MATERIAL Y MÉTODO}

Estudio descriptivo que se aborda desde el paradigma interpretativo, que utilizó metodología cualitativa y análisis de contenido. Se planteó responder a la interrogante: ¿Cuáles fueron las opiniones de padres y madres de familias vulnerables que participaron en el programa piloto "Sembrando Sonrisas" en la Región de Atacama en el año 2017?

Se siguió a Gibbs ${ }^{(19)}$ para establecer un marco de ideas temáticas que dieran origen a categorías emergentes. Se organizaron datos de acuerdo a categorías abiertas basadas en la frecuencia de citas, y el equilibrio en razón de la orientación favorable o desfavorable de las categorías.

Se utilizó como criterios de elegibilidad jardines, y luego familias, cuyo nivel de vulnerabilidad fuera muy alto, según el Registro Social de Hogares del Ministerio de Desarrollo Social y Familia. Se analizó una muestra intencionada de 194 familias que opinaron a través de un cuestionario autoaplicado, incorporado en un libro que se entregó a doce jardines públicos repartidos en cuatro comunas diferentes de la región.

El libro fue preparado por el equipo de promoción del Ministerio de Salud (MINSAL), con lenguaje pedagógico simplificado. Incluía juegos, dibujos y otras actividades para hacer atractivo el formato para las familias, incluyendo una parrilla de preguntas abiertas que consideraron creencias familiares sobre autocuidado bucal, cuidado e higiene dental y prácticas culturales relacionadas con salud bucal, analizadas en este trabajo. Las familias consintieron participar en el pilotaje del programa "Sembrando Sonrisas" y no se excusó ninguna de ellas, sin embargo, se informó de la pérdida de un libro. No se registraron datos sensibles como nombres o direcciones.

El rigor consideró los criterios de credibilidad y fiabilidad en el manejo de los datos, transcripción de respuestas íntegramente, digitalización, anonimización y almacenamiento, con resguardo exclusivo, para este trabajo, en equipos con restricción de ingreso. La transcripción de los cuadernos fue realizada por el propio equipo de investigación interdisciplinario, mientras que la triangulación entre investigadores se realizó, primero, considerando categorización independiente de dos investigadores con experiencia en metodología cualitativa (Doctor en Enfermería y Sociólogo con Magíster en Salud Pública) para, posteriormente, hacer un proceso consensuado ${ }^{(20)}$. La triangulación de grupo fue con el asesor odontológico de la Autoridad Sanitaria, para afinar la interpretación propuesta en seis sesiones presenciales con todo el equipo de trabajo, considerando su experticia en el tema y su participación en todo el proceso de planificación y aplicación del libro viajero.

En la primera fase de análisis se construyeron 25 categorías in vivo a través de un microanálisis de los datos (lectura línea por línea), para luego realizar una segunda fase, de refinamiento teórico, de donde emergieron ocho categorías teóricas como eje para discutir los hallazgos. El análisis de contenido se realizó con apoyo del programa Atlas. $\mathrm{ti}^{\oplus}$ v.8, favoreciendo con ello una interpretación integrada y teórica ${ }^{(13)}$.

Se solicitó dispensación de consentimiento a un Comité de Ética externo, dado que los datos son resultado de una evaluación realizada previamente, 
en donde no intervino el equipo que realizó el análisis. Este aspecto, limitó en parte el nivel de saturación de algunas categorías desde la perspectiva de los investigadores, sin embargo, muestra de forma natural cuáles son los aspectos que más interesan a las familias en el ámbito de la salud bucal de sus hijos(as). Además, el uso del cuaderno, por un periodo de un año en cada nivel, generó una importante acumulación de información basada, principalmente, en la motivación de cada familia que permitió contar con gran cantidad de datos para crear categorías teóricas, suficientemente saturadas de información (frecuencia y equilibrio) para construir el análisis.

\section{RESULTADOS}

Las edades de los niños/as incluyeron lactantes menores, mayores y preescolares, todos menores de seis años, y asistentes a jardines públicos con alto riesgo social, que se caracterizan por dar cobertura a familias en situación de pobreza multidimensional, baja escolaridad, dificultades para lograr cobertura sanitaria, empleo informal y precariedad en la vivienda.

A partir del proceso de análisis de contenido, a continuación se describen las ocho categorías teóricas vinculadas a los datos como fuente empírica de la interpretación:

1) Hábitos que favorecen la salud bucal: Los padres en su mayoría mencionaron utilizar en sus hijos(as) cepillo de dientes y pasta: "Siempre como familia llevamos en cada viaje o paseo, nuestros útiles de aseo, incluyendo el cepillo de dientes y pasta dental, nos lavamos en cada comida" (F196). Además, mencionan consumo de alimentos saludables: "Preferimos frutas frescas, yogurt y leche, en vez de comida chatarra" (F68). Con menos frecuencia se mencionaron jugos y bebidas en vez de agua y galletas: "Para un paseo echamos papas fritas, jugo de naranja, galletas, cepillo de dientes, shampoo y jabón" (F44). Llama la atención que algunas familias utilizaban enjuagatorio bucal e hilo dental con sus hijos(as): "No olvidamos nada, siempre llevamos cepillo, pasta, enjuagatorio bucal e hilo dental para los niños"(F65), siendo infrecuente su uso en niños(as) por los riesgos asociados a la salud.

2) Actitudes de los padres sobre la salud bucal de sus hijos(as): En sentido positivo, las familias reconocen su compromiso por mejorar y favorecer la salud bucal de sus hijos(as): "nos comprometemos a mejorar el lavado de los dientes con más tiempo y empezar a enseñar que la leche se toma en vaso" (F114). En sentido negativo, se describen actitudes distantes con los hábitos bucales de sus hijos, y se les asigna mayor responsabilidad sobre cuidado bucal del que pueden asumir como lactantes o preescolares: "se queda dormida y aún toma mamadera por las noches" (F50), "siempre consume alimentos con azúcar" (F51), "es flojita para el lavado de dientes" (F36).

3) Cambio en los estilos de vida y cuidado dental: Las familias reconocen un mayor acceso al conocimiento y recursos para el cuidado dental que en el pasado: "antes no estaban todos los productos que existen ahora y el hábito de cepillado no era tan frecuente" (F50). Mencionan además, creencias y costumbres transmitidas en entornos familiares y éstas no siempre responden a prácticas saludables: "se lavaba con bicarbonato" (F1), "refregándose con carbón" (F110, F131), "masticando una ramita de sauce u hojas de té" (F116), "con carbón en polvo, ceniza y zumo de limón o mezcla de hojas de tabaco y miel" (F84).

4) Conocimiento sobre salud bucal de padres y madres: Las familias reconocen la importancia del cuidado bucal y su relación con el estado general de salud: "No debemos olvidar la importancia del cuidado bucal y corporal, manteniendo nuestros dientes limpios" (F121). Se valoran avances en el acceso a la atención odontológica convencional: "se accede a controles con los dentistas por intermedio del consultorio o particular, y hay más recursos como para hacerse tratamientos" (F183).

5) Importancia de la dentición decidua: Las familias describieron algunas ideas erróneas sobre la dentición temporal: "durante toda su vida tendrán dientes de leche" (F4), "porque son los dientes más sanos y fuertes y es dificil que se le piquen" (F47).

6) Cambios de hábitos relacionados con salud bucal: La mayoría de las familias describieron hábitos que favorecen la salud bucal, como el uso del kit de limpieza, alimentación saludable e importancia de la higiene bucal en los niños(as); esto se relaciona con la adquisición de conocimientos en salud bucal: "El lavado es constante, ya que tienen el hábito" (F153), "Ya no usa mamadera y aunque a 
veces tenga sueño han sido pocas las veces que se ha dormido sin lavar" (F174).

7) Relación lúdica con la higiene bucal: Las familias destacaron uso de técnicas lúdicas en la enseñanza de hábitos en niños(as), acompañando o demostrando cómo realizar la técnica de cepillado: "tratamos de corregir si no lo están haciendo bien, con demostraciones y que sea divertido" (F183), "que sea un hábito entretenido" (F164).

8) Relación entre la imagen física y la salud bucal: Las familias mencionaron preocupación por mantener una sonrisa sana y la valoran debido a la apariencia física: "nuestros dientes son muy importantes para tener una sonrisa linda" (F84, F89, F92, F93, F155).

\section{DISCUSIÓN}

Los establecimientos que participaron de la estrategia, dan cobertura educativa a niños(as) provenientes de familias con altos niveles de vulnerabilidad socioeconómica, situación que, como se ha mencionado, se relaciona con bajos niveles de salud en la población infantil ${ }^{(11,21)}$. Chile ha favorecido políticas públicas desde el enfoque multidimensional desarrolladas en favor de la infancia, permitiendo un abordaje integral, desde la institucionalidad y desde la complejidad de las desigualdades sociales, priorizando intervenir a los más desfavorecidos ${ }^{(22)}$.

Las familias tienen claridad en cuanto a que la adquisición de hábitos bucales saludables supone una tendencia a repetir y reproducir ciertas acciones de la misma forma y bajo las mismas circunstancias. Este tipo de conocimiento organizado es resultado del condicionamiento simple. Estudios en escolares realizados por Loewenstein, Price y Volpp ${ }^{(23)}$ sugieren que la formación de hábitos saludables debe comenzar tempranamente en la infancia, utilizando estímulos positivos, porque así es más fácil que estos puedan mantenerse en el tiempo.

En este sentido, la estrategia está dirigida a familias en un contexto comunitario, considerando la participación de los padres en el hogar, y educadores en el jardín infantil, ampliando sus propias competencias para realizar mejores acciones en el desarrollo de hábitos dentales. La importancia de intervenciones preventivas y de educación en espacios significativos, como el entorno familiar y escolar, han sido ampliamente reportadas ${ }^{(24,25)}$. En este aspecto, las opiniones de los padres en el cuaderno viajero se orientan favorablemente hacia el cuidado dental, lo que demuestra que acciones promocionales, apoyadas permanentemente por el equipo de salud bucal primario, tienen un impacto positivo en la actitud de los padres y en la formación de hábitos en los niños(as). En el entorno familiar el autocuidado del niño/a es fundamental: se elige la alimentación, el consumo de agua, las rutinas en el uso de la mamadera, las costumbres para desestimar el uso de chupetes o no besar en la boca; y la literatura es coincidente en señalar que, especialmente la actitud de la madre contribuye a que prácticas saludables se adquieran en etapas tempranas de la infancia ${ }^{(26-28)}$.

La estrategia de participación comunitaria se escapa a la tradición paternalista del modelo biomédico, permitiendo que la familia sea responsable del cuidado de la salud bucal de sus hijos(as), mientras el jardín y equipo de salud actúan como facilitadores del proceso. En esta acción colaborativa, se genera una comunicación que permite, según Busse y Godoy ${ }^{(29)}$, "informar, influir y motivar a las familias sobre temas de salud relevantes". El empoderamiento de los padres se relaciona fundamentalmente con el conocimiento en salud, el que juega un rol crítico en cualquier cambio de conducta, afectando de manera positiva el acceso a la atención médica, resultados de salud y costos sanitarios ${ }^{(10-17)}$. El aumento del conocimiento en salud dental en niños(as) es el resultado de estrategias en los colegios, cuando aún pueden moldearse conductas en el entorno natural de los/as niños(as) ${ }^{(30)}$. Como el conocimiento sobre salud bucal se relaciona directamente con un mejor nivel de salud bucal, países desarrollados han implementado fuertes estrategias de promoción y prevención, basada en la educación individual y comunitaria, incorporando conocimientos sobre el cepillado dental, uso de fluoruros en pastas dentales y alimentación saludable con bajo consumo de alimentos cariogénicos ${ }^{(5,26,31)}$. Junto con esto, la importancia de promover las visitas preventivas al odontólogo y al control de supervisión de salud, han demostrado ser estrategias relevantes para mejorar los indicadores de daño en la población infantil ${ }^{(16)}$.

En los discursos, se siguen encontrando algunas 
ideas equivocadas con respecto a la dentición decidua, por lo que es importante aclarar que cumplen una función vital para el futuro de la salud bucal de los niños(as), y reforzar que deben ser conservados $\operatorname{sanos}^{(32)}$. Siguiendo la misma idea, algunas familias mencionan el uso de seda dental y enjuagatorios bucales en niños(as), lo que implica riesgos cuando no existe una debida supervisión. Quizás las familias podrían estar siendo influenciadas por el amplio y llamativo mercado de productos dentales, sin control sanitario y que, sin la debida orientación, pueden ser un riesgo para la salud de la población infantil ${ }^{(33)}$. El uso de seda dental requiere que el niño(a) tenga niveles de madurez cognitiva y motricidad adecuados y la posibilidad de sufrir accidentes al usar enjuagatorios bucales es suficiente para considerarlo poco apropiado en ellos(as).

En el cuidado bucal, los aspectos socioculturales deben ser considerados por el equipo sanitario, ya que estos influyen en las elecciones familiares ${ }^{(28)}$.

Algunas familias mencionaron el uso de bicarbonato, cenizas (lejía), limón o yerbas, para la limpieza dental y estas prácticas se relacionan con el estudio realizado sobre hábitos bucales en población mapuche, en donde el $62 \%$ de las personas encuestadas utilizaba remedios tradicionales para sanar sus problemas dentales ${ }^{(34)}$. Estas prácticas ancestrales pueden provocar abrasión del esmalte, no siendo recomendables en ningún caso para niños(as) o adultos. Por ello, la revisión de estas prácticas de cuidado deben ser analizadas con cada familia, diferenciando aquellas saludables de las que no. De esta forma se propicia el desarrollo de capacidades para el autocuidado con pertinencia cultural, y evitar riesgos para la salud bucal de la familia.

Si bien, la mayoría de las familias refieren adherir a la indicación de postergar el consumo de azúcar y aunque la relación entre alimentación y enfermedades dentales ha sido ampliamente documentada, en especial el consumo de azúcares y la aparición de caries en la infancia, algunas familias reconocen la costumbre de incorporarla tempranamente. En este sentido, reforzar una alimentación saludable y regular el consumo de alimentos azucarados y cariogénicos en el hogar, es un contenido educativo fundamental para mantener la salud bucal ${ }^{(11)}$. Los padres cuidadores deben actuar en consecuencia para guiar y ser modelos para sus hijos(as), evitando el castigo en todas sus formas, ser persistente en el cepillado, establecer reglas, rutinas diarias y propiciar entornos agradables ${ }^{(5)}$. Un niño/a lactante es totalmente dependiente del cuidado dental, mientras que un preescolar podrá realizar la higiene con autonomía, pero supervisado por un adulto. La estrategia, en este sentido, mostró que las etapas de desarrollo infantil no siempre son de conocimiento de la familia, por lo que considerar contenidos educativos relacionados con la madurez y desarrollo psicomotor de los niños(as) es fundamental ${ }^{(35)}$. Los cambios de conducta son progresivos y secundarios a la etapa de conocimiento y sensibilidad por parte de la familia ${ }^{(25)}$.

En este contexto, un ambiente de juego ha sido reportado por los padres para facilitar experiencias de aprendizaje en los nińos(as), respetando su entorno cultural. Los beneficios del juego en la adquisición de hábitos bucales favorece el desarrollo sensorial, la comprensión del mundo, la autoeficacia, la interacción social y el aprendizaje de roles, por lo que el juego es una herramienta educativa a considerar en los programas de salud infantil $^{(36)}$. Los jardines infantiles juegan un rol importante porque aportan con dinámicas lúdicas a través de la planificación didáctica, y cumplen una función esencial en la entrega de conocimiento sanitario a los niños(as).

En la última categoría se observa que los padres relacionan la dentición con la imagen corporal, lo que tiene alcances en lo personal, social y cultural. En el mundo occidental una bella sonrisa simboliza juventud, salud y felicidad, por lo que estar desdentado y perder estos símbolos, provoca un duelo, similar al que ocurre cuando se pierde alguna otra parte del cuerpo. Por consiguiente, una salud bucal deficiente afectará negativamente a personas jóvenes, siendo la imagen estética la principal razón por la que el adolescente busca atención odontológica. Cuando la imagen social se ve perjudicada, se afecta su autoestima que interfiere en sus relaciones personales y vida social ${ }^{(37)}$.

Finalmente, este primer acercamiento a las opiniones de familias vulnerables sobre la salud bucal en la infancia releva la importancia de la familia como base para el autocuidado de sus miembros, y visibiliza las fortalezas de la estrategia. Esta podría ser replicada en distintas regiones del país, y adaptarse a distintas realidades considerando características particulares de cada población. 
La principal limitación de esta investigación dice relación con la falta de acuerdo intersubjetivo que se da habitualmente entre los investigadores cualitativos y los sujetos de estudio, ya que los datos fueron entregados luego de ejecutada la estrategia. Esto disminuyó la posibilidad de tomar decisiones muestrales, hacer ajustes metodológicos y otros aspectos propios del enfoque cualitativo.

\section{CONCLUSIONES}

Es posible concluir que las familias que participaron en el programa de promoción de la salud bucal "Sembrando Sonrisas" en la Región de Atacama, en el año 2017, centraron sus opiniones en ocho categorías teóricas: hábitos y actitudes que favorecen la salud bucal de sus hijos(as), cambio en los estilos de vida y cuidado dental, conocimiento sobre

\section{REFERENCIAS}

1. Giacaman RA, Bustos IP, Bravo VL, Mariño RJ. Impact of rurality on the oral health status of 6-year-old children from central Chile: The Epi Maule study. Rural Remote Health [Internet]. 2015 [citado 8 dic 2019]; 15(3135): 1-9. Disponible en: https://www.rrh.org.au/journal/article/3135

2. Ministerio de salud de Chile. Plan nacional de salud bucal 2018-2030 [Internet]. Santiago, Chile: Ministerio de Salud. 2017 [citado 15 dic 2019]; Disponible en: https://www.minsal.cl/wp-content/ uploads/2017/12/Plan-Nacional-Salud-Bucal2018-2030-Consulta-Pública-20_12_2017.pdf

3. Saliba GC, Barreto SG, Moraes DF, Íspes GA, Moreira AR. Oral health education in school: parents' attitudes and prevalence of caries in children. Rev odontol UNESP [Internet]. 2015 [citado 24 jun 2020]; 44 (5): 285-291. Disponible en: https://doi.org/10.1590/1807-2577.0097

4. Nguyen TT, Tien BB, Nguyen MS, Olak J, Saag M. Effect of School Oral Health Promotion Programme on dental health and health behaviour in Vietnamese schoolchildren. Pediatric Dental Journal [Internet]. 2016 [citado 24 jun 2020]; 26(3): 115-121. Disponible en: https://doi. org/10.1016/j.pdj.2016.09.001

5. Casamassimo P, Hammersmith K, Gross E, Amini salud bucal de padres y madres, importancia de la dentición decidua, cambios de hábitos relacionados con salud bucal, relación lúdica con la higiene bucal y relación entre la imagen física y la salud bucal.

Estas categorías reafirman el papel esencial de la promoción de la salud y la prevención de la enfermedad bucal en el contexto cotidiano de las familias, por lo que estas áreas sustantivas identificadas pueden ser utilizadas tanto para mejorar los contenidos educativos de la estrategia como para permitir adaptarla a realidades diversas.

\section{AGRADECIMIENTOS}

Al Dr. Luis González Araya Asesor Odontológico de la Seremi de Salud Atacama, quien acompañó el proceso como Asesor Regional de Salud Bucal.

H. Infant Oral Health. An Emerging Dental Public Health Measure. Dent Clin N Am [Internet]. 2018 [citado 28 oct 2019]; 62(2): 235-244. Disponible en: https://doi.org/10.1016/j.cden.2017.11.004

6. Verdugo LS, García CR, Del Castillo ED. Calidad de vida relacionada a la salud oral en escolares del sector urbano y rural. Rev Estomatol Herediana [Internet]. 2018 [citado 15 abr 2019]; 28(2): 105114. Disponible en: https://doi.org/10.20453/reh. v28i2.3326

7. Santi MF. Vulnerabilidad y ética de la investigación social: perspectivas actuales. Rev Latinoam Bioet [Internet]. 2015 [citado 10 feb 2019]; 15(2): 5273. Disponible en: http://www.scielo.org.co/pdf/ rlb/v15n2/v15n2a05.pdf

8. Colombo S, Ferrazzano GF, Beretta M, Paglia L. Dental caries prevention: a review on the use of dental sealants. Italian Journal of Dental Medicine [Internet]. 2018 [citado 15 nov 2019]; 3-4(0): 8186. Disponible en: http://www.dentalmedjournal. it/files/2018/12/JDM_2018_004_INT@081-086. pdf

9. Arteaga O, Urzúa I, Espinoza I, Muñoz A, Mendoza C. Prevalence of Caries and Tooth Loss in Population Aged 65 to 74 in Santiago, Chile. Rev Clin Periodoncia Implantol Rehábil Oral [Internet]. 2009 [citado 02 dic 2019]; 2(3): 161166. Disponible en: https://doi.org/10.1016/ 
S0718-5391(09)70027-8

10. Tellez M, Zini A, Estupiñan-Day S. Social Determinants and Oral Health: An Update. Dental public health. Curr Oral Health Rep [Internet]. 2014 [citado 10 abr 2019]; 1(0): 148-152. Disponible en: https://doi.org/10.1007/s40496014-0019-6

11. Spencer N, Raman S, O'Hare B, Tamburlini G. Addressing inequities in child health and development: towards social justice. BMJ Paediatr Open [Internet]. 2019 [citado 15 ene 2020]; 3(1): e000503. Disponible en: https://doi.org/10.1136/ bmjpo-2019-000503

12. Mouradian WE, Wehr JD, Crall JJ. Disparities in Children's Oral Health and Access to Dental Care. JAMA [Internet]. 2000 [citado 10 dic 2019]; 284(20): 2625-2631. Disponible en: https://doi. org/10.1136/bmjpo-2019-000503

13. Ruiz DC, Morales C. Social determination of the oral health-disease process: a social-historical approach in four Latin American countries. Invest Educ Enfer [Internet]. 2015 [citado 11 dic 2019]; 33(2): 248-259. Disponible en: http://dx.doi. org/10.17533/udea.iee.v33n2a07

14. Povlsen L, Borup I. Health Promotion: A developing focus area over the years. Scandinavian Journal of Public Health [Internet]. 2015 [citado 18 may 2019]; 43(16): 46-50. Disponible en: https://doi. org/10.1177/1403494814568595

15. Lee JY, Divaris K. The Ethical Imperative of Addressing Oral Health Disparities: A Unifying Framework. Jour Dent Research [Internet]. 2014 [citado 14 feb 2020]; 93(3): 224-230. Disponible en: $10.1177 / 0022034513511821$

16. Fresno MC, Jeldes G, Estay J, Martin J. Prevalencia, severidad de caries dental y necesidad de tratamiento restaurador en escolares de 6 a 12 Ańos de la Provincia de Santiago, Región Metropolitana. Rev Clin Periodoncia Implantol Rehabil Oral [Internet]. 2019 [citado 3 dic 2019]; 12(2): 81-86. Disponible en: http://dx.doi.org/10.4067/S071901072019000200081

17. Cartes RA. Alfabetismo en salud: bases conceptuales y evidencia en odontología. Medisan [Internet]. 2015 [citado 14 feb 2019]; 19(4): 562-570. Disponible en: http://scielo.sld.cu/pdf/san/v19n4/ san13194.pdf

18. Riadi CC, Escalona LX, Avalos PL, Díaz NV. Evaluación del efecto del Plan de Salud Oral de embarazadas en sus hijos en Lo Barnechea, Chile. Odontoestomatología [Internet]. 2015 [citado 24 ago 2020]; 17(26): 24-34. Disponible en: http:// www.scielo.edu.uy/pdf/ode/v17n26/v17n26a04. pdf

19. Gibbs G. El análisis de datos cualitativos en investigación cualitativa. $1^{\text {era }}$ Ed. Madrid: Morata; c2012. 63-76 p.

20. Morse JM. Critical Analysis of Strategies for Determining Rigor in Qualitative Inquiry. Qualitative Health Research [Internet]. 2015 [citado 10 nov 2019]; 25(9): 1212-1222. Disponible en: https://doi.org/10.1177/1049732315588501

21. Cançado M, Wisniewski f, Correa T, Vaz J, Pereira E, Concha X. Salud bucal e indicadores socioeconómicos de adolescentes residentes en una región de extrema pobreza. Rev Fac Odontol Univ Antioq [Internet]. 2018 [citado 21 ene 2020]; 29(2): 311-328. Disponible en: http://dx.doi. org/10.17533/udea.rfo.v29n2a4

22. Bredregal P, Torres A, Carvallo C. Chile Crece Contigo: el Desafío de la Protección Social a la Infancia. Programa de las naciones unidas para el desarrollo [Internet]. Santiago, Chile: Organización Naciones Unidas; 2014 [citado 14 oct 2019]. Disponible en: https://www.undp.org/content/dam/ chile/docs/pobreza/undp_cl_pobreza_cap5_chile_ crece.pdf

23. Loewenstein G, Price J, Volpp K. Habit formation in children: Evidence from incentives for healthy eating. J Health Econ [Internet]. 2016 [citado 14 oct 2019]; 45(0): 47-54. Disponible en: https:// doi.org/10.1016/j.jhealeco.2015.11.004

24. De Castilho AR, Mialhe FL, Barbosa TS, Puppin RP. Inuence of family environment on children's oral health: a systematic review. J Pediatr [Internet]. 2013 apr-march [citado 23 nov 2019]; 89(2): 116-123. http://dx.doi.org/10.1016/j. jped.2013.03.014

25. Whitebread D, Bingham S. Habit formation and learning in young children. University of Cambridge [Internet]. 2013 [citado 24 nov 2019]. Disponible en: https://mascdn.azureedge.net/cms/ the-money-advice-service-habit-formation-andlearning-in-young-children-may2013.pdf

26. Kizito A, Caitlin M, Wang Y, Kasangaki A, Macnab A. Oral health promotion in schools: rationale and evaluation. Health Education [Internet]. 2014 [citado 10 nov 2019]; 114(4): 293-303. Disponible en: http://dx.doi.org/10.1108/HE-08-2013-0042

27. Sehrawat P, Shivlingesh KK, Gupta B, Anand R, Sharma A, Chaudhry M. Oral health knowledge, awareness and associated practices of pre-school children's mothers in Greater Noida, India. Niger Postgrad Med J [Internet]. 2016 [citado 21 nov 2019]; 23(3): 152-157. Disponible en: http:// www.npmj.org/text.asp?2016/23/3/152/190344

28. Shetty RM, Deoghare A, Rath S, Sarda R, Tamrakar A. Influence of mother's oral health care knowledge on oral health status of their preschool child. Saudi J Oral Sci [Internet]. 2016 [citado 09 nov 
2019]; 3(1): 12-16. Disponible en: http://dx.doi. org/10.4103/1658-6816.174291

29. Busse P, Godoy S. Comunicación y salud. Cuadernos info [Internet]. 2016 [citado 09 may 2019]; 38(0): 10-13. Disponible en: https://scielo.conicyt. cl/pdf/cinfo/n38/art01.pdf

30. Blaggana A, Grover V, Kapoor A, Blaggana V, Tanwar R, Kaur H, et al. Oral Health Knowledge, Attitudes and Practice Behaviour among Secondary School Children in Chandigarh. J Clin Diagn Res [Internet]. 2016 [citado 14 dic 2019]; 10(10): ZC01-ZC06. Disponible en: https://dx.doi. org/10.7860\%2FJCDR\%2F2016\%2F23640.8633

31. Jadue LH, Delgado BI, Simian MM, Prieto UF, Huberman CJ. Programa de Salud Oral Integral para Niños(as) de 6 años como Problema de Salud con Garantía Explícita en Chile: La Participación de los Pediatras. Rev Chil Pediatr [Internet]. 2009 [citado 10 feb 2019]; 80(6): 534-538. Disponible en: http://dx.doi.org/10.4067/S037041062009000600006

32. Torres A, López FB, Parra V, Vázquez C, Segura PS, Cetin Z, et al. Chile Crece Contigo: Implementation, results, and scaling-up lessons. Child Care Health Dev [Internet]. 2017 [citado 22 dic 2019]; 44(1): 4-11. Disponible en: https://doi. org/10.1111/cch.12519

33. Martínez MP. The use of fluoride dentifrices in children: conceptual bases in a confusing context.
A topic review. Rev Fac Odontol Univ Antioq [Internet]. 2017 [citado 14 dic 2019]; 29(1): 187210. Disponible en: http://dx.doi.org/10.17533/ udea.rfo.v29n1a10

34. Misrachi CL, Manríquez JU, Fajreldin VCh, Kuwahara KA, Verdaguer CM. Creencias, conocimientos y prácticas en salud oral de la población Mapuche-Williche de isla Huapi, Chile. Rev Fac Odontol Univ Antioq [Internet]. 2014 [citado 2 feb 2019]; 25(2): 342-358. Disponible en: http:// www.scielo.org.co/pdf/rfoua/v25n2/v25n2a08.pdf

35. Cabrera BC, Dupeyrón MN. El desarrollo de la motricidad fina en los niños y niñas del grado preescolar. Rev Mendive [Internet]. 2019 [citado 02 feb 2019]; 17(2): 222-239. Disponible en: http://scielo.sld.cu/pdf/men/v17n2/1815-7696men-17-02-222.pdf

36. Cáceres FZ, Granada MA, Pomés MC. Inclusión y Juego en la Infancia Temprana. Rev latinoam educ inclusiva [Internet]. 2018 [citado 4 feb. 2019]; 12(1): 181-198. Disponible en: https://dx.doi. org/10.4067/S0718-73782018000100181

37. Goulart M, Condesa AM, Hilbert JB, Hugo FN, Celeste RK. Concerns about dental aesthetics are associated with oral health related quality of life in Southern Brazilian adults. Ciênc Saúde Coletiva [Internet]. 2018 [citado 18 nov 2019]; 23(11): 3957-3964. Disponible en: http://dx.doi. org/10.1590/1413-812320182311.24172016 